Acta Crystallographica Section B

Structural

Science

ISSN 0108-7681

\section{High-pressure neutron diffraction study of L-serine-I and L-serine-II, and the structure of L-serine-III at 8.1 GPa}

The hydrostatic compression of L-serine- $d_{7}$ has been studied to $8.1 \mathrm{GPa}$ by neutron powder diffraction. Over the course of this pressure range the compound undergoes two phase transitions, the first between 4.6 and $5.2 \mathrm{GPa}$, yielding Lserine-II, and the second between 7.3 and $8.1 \mathrm{GPa}$, yielding Lserine-III. All three polymorphs are orthorhombic, $P 2_{1} 2_{1} 2_{1}$, and feature chains of serine molecules connected via head-totail ND...O hydrogen bonds formed between ammonium and carboxylate groups. The chains are linked into a ribbon by a second set of ND ...O hydrogen bonds. The hydroxyl moieties are distributed along the outer edges of the ribbon and in phase I they connect the ribbons into a layer by chains of OD ...OD hydrogen bonds. The layers are connected together by a third set of ND ... O hydrogen bonds, forming $R_{4}^{3}(14)$ rings with substantial voids at their centres. In the transition from phase I to II these voids begin to close up, but at the cost of breaking the OD ...OD chains. The OD ...OD hydrogen bonds are replaced by shorter OD ...O hydrogen bonds to carboxylate groups. At $7.3 \mathrm{GPa}$ the $\mathrm{O} \cdots \mathrm{O}$ distance in the OD ... O hydrogen bonds measures only 2.516 (17) $\AA$, which is short, and we propose that the phase transition to phase III that occurs between 7.3 and $8.1 \mathrm{GPa}$ relieves the strain that has built up in this region of the structure. The hydroxyl D atom now bifurcates between the OD ...O contact that had been present in phase II and a new OD . . O contact formed to a carboxylate in another layer. Hirshfeld surface fingerprint plots show that D ...D interactions become more numerous, while hydrogen bonds actually begin to lengthen in the transition from phase II to III.

\section{Introduction}

Pressure is an important thermodynamic variable in the study of molecular systems as it provides a useful tool for changing the hierarchy of interactions in the solid state. For example, hydrogen-bonding interactions are usually regarded as being 'structure-directing', but as pressure increases the need to pack molecules efficiently is expected to begin to assume more importance. The application of pressure, unlike temperature, also allows structural studies to be performed that depend on changes in volume alone, and not the simultaneous effects of volume and thermal energy (Fourme et al., 2001).

The importance of pressure is well known in marine biology and deep-sea diving physiology. The application of pressure to biological systems has been known to have major effects, from the deactivation of enzymes (Somero, 1992) to killing bacteria (Johnson et al., 1974). In fact, the structural effects of pressure were observed in proteins by Bridgeman as early as 1914,
Received 23 November 2005

Accepted 15 May 2006
(C) 2006 International Union of Crystallography Printed in Great Britain - all rights reserved 
when the white of an egg subjected to hydrostatic pressure at room temperature became coagulated in appearance, becoming much like a hard-boiled egg. Recent work by Fourme et al. (2001) has opened up the possibility of collecting high-resolution, high-pressure data on large macromolecules using short wavelength X-rays ( $c a 0.3 \AA$ ). More recently, the first crystal structure of a macromolecular assembly under high-pressure was published on the structure of cubic cowpea mosaic virus crystals (Girard et al., 2005).

A recent goal in the field of small-molecule crystallography has been to gain an understanding of the changes which occur in molecular systems as a function of pressure at atomic resolution (Boldyreva, 2004; Katrusiak, 2004; Hemley \& Dera, 2000). Amino acids have several advantages in this area, as they are polyfunctional and form well defined intermolecular interactions such as hydrogen bonds and dipolar contacts; the results also have some relevance to the macromolecular work described above. In recent years a number of high-pressure crystallographic studies have been carried out on amino acids and related compounds, including glycine (Dawson et al., 2005; Boldyreva et al., 2003), cystine (Moggach, Allan, Parsons, Sawyer \& Warren, 2005) and cysteine (Moggach et al., 2006).

We recently described the effect of pressure on the anhydrous orthorhombic phase of L-serine, L-serine-I, which undergoes a single crystal to single crystal phase transition at 5.4 GPa to a new high-pressure phase, L-serine-II (Moggach, Allan, Morrison, Parsons \& Sawyer, 2005). This transition occurs with no change in symmetry. The reduction in volume is achieved by the compression of interstitial voids. In addition, the strain present in some hydrogen-bonded interactions, which become very short just below the transition pressure, is also relieved during the phase transformation.

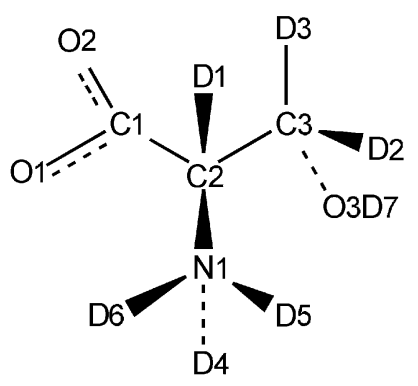

Our previous study on serine was carried out using X-ray diffraction and certain inferences regarding $\mathrm{H}$-atom positions were made on the basis of $\mathrm{N}$ and $\mathrm{O}$ positions. We now describe a study of the compression of L-serine by neutron powder diffraction, which enables statements on $\mathrm{H}$-atom positions to be made with a greater degree of certainty. Recently, a second phase transition was observed in L-serine via a high-pressure Raman study, the transition taking place at 7.8 GPa (Kolesnik et al., 2005). Here we confirm this reported transition to a third high-pressure phase of serine, L-serine-III, at $8.1 \mathrm{GPa}$, and describe its structure for the first time.

\section{Experimental}

L-Serine- $d_{7}(98.2$ atom $\%$ D) was purchased from CDN isotopes (catalogue number D-6466). One small crystallite was removed directly from the sample bottle and shown to be Lserine-I by single-crystal diffraction (Kistenmacher et al., 1974). The absolute configuration at the $\alpha$-carbon atom was taken as $S$.

\subsection{Neutron powder diffraction at high pressure}

Ambient-temperature, high-pressure neutron powder diffraction data were collected by the time-of-flight technique at the PEARL beamline high-pressure facility (HiPr) at ISIS. Approximately $50 \mathrm{~mm}^{3}$ of L-serine- $d_{7}$ was gently ground into a fine powder and loaded into a Paris-Edinburgh (P-E) highpressure cell. The $\mathrm{P}-\mathrm{E}$ cell ram pressure was monitored and controlled by means of a computer-controlled hydraulic system. The sample was loaded into a null-scattering $\mathrm{Ti}-\mathrm{Zr}$ alloy capsule gasket (Marshall \& Francis, 2002). A pellet of lead was included as a pressure marker.

Time-of-flight neutron powder diffraction data suitable for structure refinement were obtained by electronically focusing the 1080 individual detector element spectra of the main PEARL/HiPr $2 \theta=90^{\circ}$ detector bank. The summed pattern was then normalized with respect to the incident beam monitor and the scattering from a standard vanadium calibration sample. Lastly, the diffraction pattern intensities were corrected for the wavelength and scattering-angle dependence of the neutron attenuation by the P-E cell anvil (WC) and gasket (TiZr) materials.

In order to ensure that the pressure applied to a crystal held in a pressure cell is uniform, it is necessary to immerse the sample in a medium which displays hydrostatic behaviour throughout the pressure range of interest. A 4:1 mixture of methanol and ethanol is commonly used in high-pressure work, although L-serine has some solubility in this mixture. A 1:1 mixture of deuterated pentane and 2-propanol was therefore used for an initial survey. Data were collected in approximately $0.7 \mathrm{GPa}$ steps from $0.1 \mathrm{GPa}$ to a final pressure of $4.3 \mathrm{GPa}$. Peak broadening occurred above $4.3 \mathrm{GPa}$, suggesting that conditions became non-hydrostatic at high pressures.

Based on our previous work on crystal growth from solutions at high pressure, the solubility of L-serine was expected to decrease at elevated pressures (Fabbiani et al., 2005). In order to extend the pressure range for this study a second sample of L-serine was loaded with a 4:1 mixture of MeOD:EtOD as the hydrostatic medium. Diffraction data were collected in approximately $1 \mathrm{GPa}$ steps from $4.6 \mathrm{GPa}$ to a final pressure of $8.1 \mathrm{GPa}$. On increasing pressure to $5.2 \mathrm{GPa}$, the phase transition from L-serine-I to L-serine-II was observed, a transition which we have observed previously in a single-crystal high-pressure $\mathrm{X}$-ray diffraction experiment on a non-deuterated sample of L-serine-I (Moggach, Allan, Morrison, Parsons \& Sawyer, 2005). On increasing pressure further to $8.1 \mathrm{GPa}$, another phase transition was observed to a 


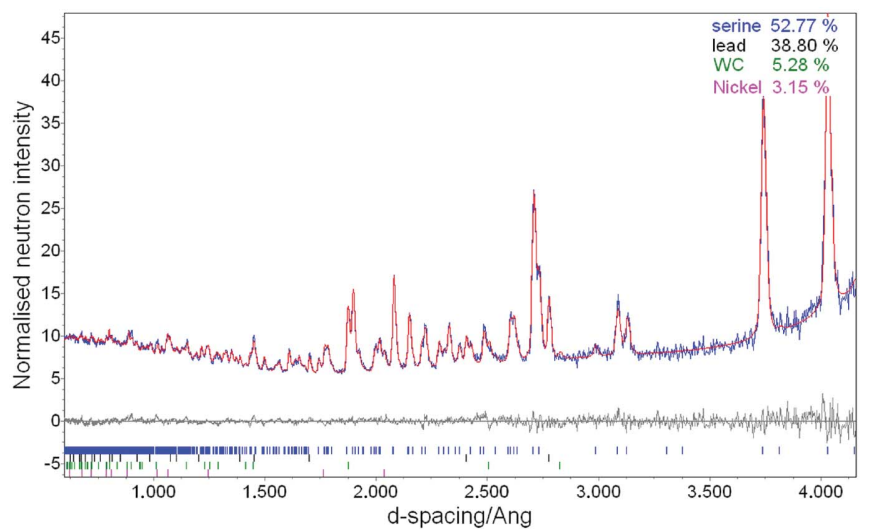

(a)

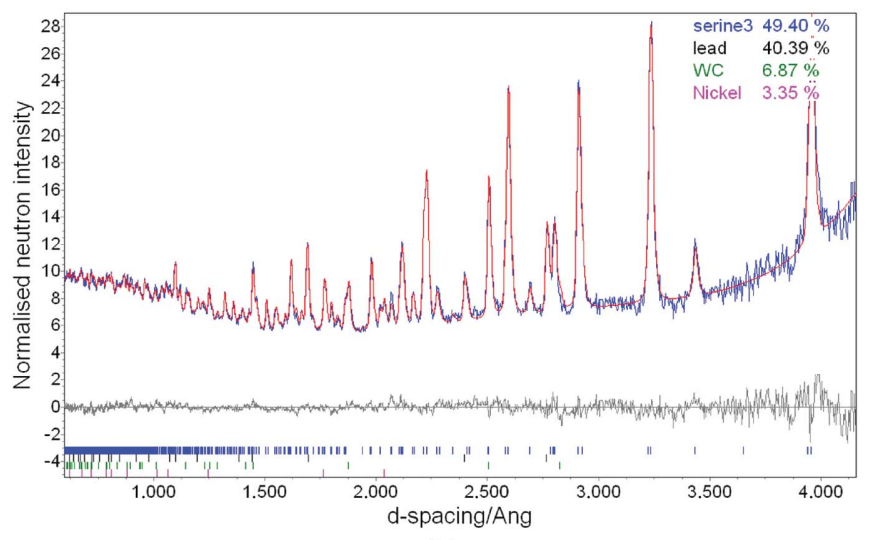

(b)

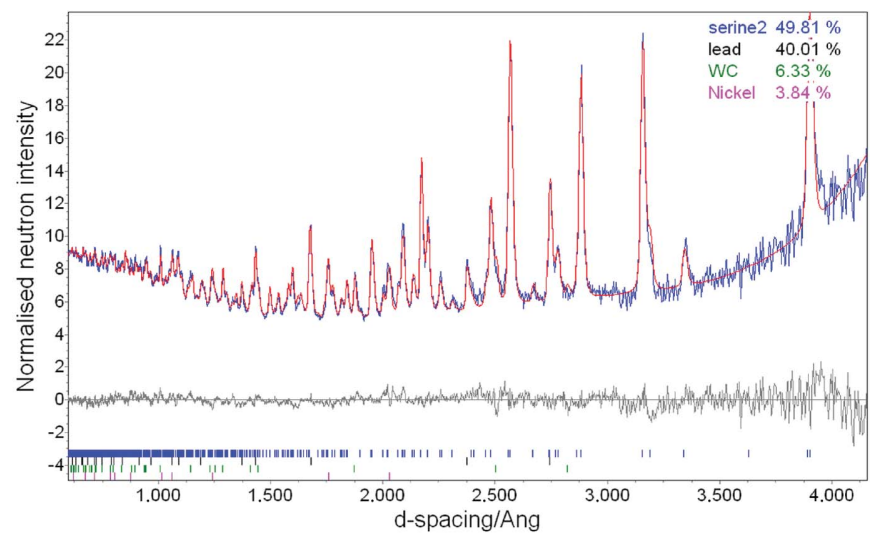

(c)

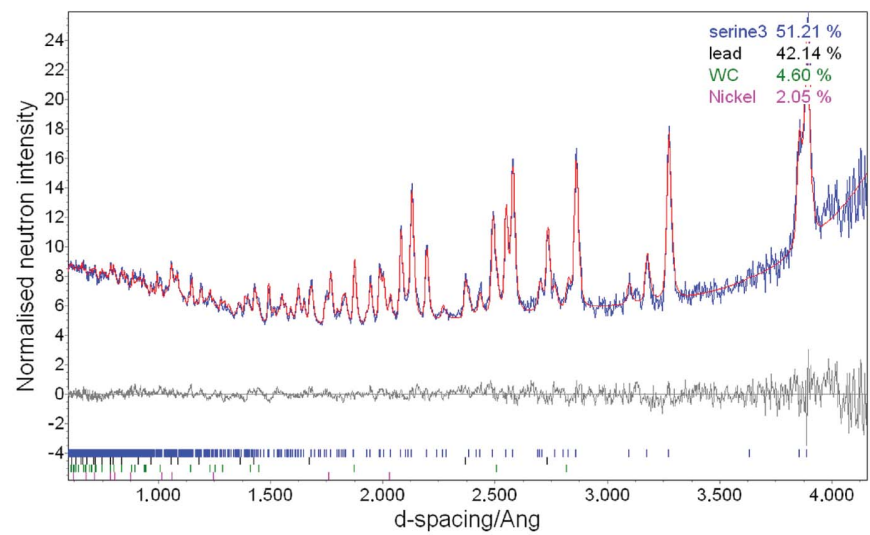

(d)

Figure 1

Rietveld refinement plots of L-serine: $(a)$ phase I at $4.6 \mathrm{GPa} ;(b),(c)$ phase II at 5.2 and $7.3 \mathrm{GPa} ;(d)$ phase III at $8.1 \mathrm{GPa}$. The blue and red lines correspond to the observed and calculated powder patterns, respectively; the difference plot is shown in grey.

hitherto uncharacterized phase of L-serine which we have designated L-serine-III.

\subsection{Structure solution and refinement}

Powder-pattern indexing, structure solution and Rietveld refinements were carried out using TOPAS-A (Coelho, 2005). Rietveld refinement of phases I and II was carried out starting from the 4.8 and $5.4 \mathrm{GPa}$ coordinates of Moggach, Allan, Morrison, Parsons \& Sawyer (2005) for L-serine-I and Lserine-II, respectively.

The structure of the new phase (L-serine-III) was indexed on an orthorhombic unit cell, $a=6.5574, b=9.5397, c=$ $5.6101 \AA$, in the space group $P 2_{1} 2_{1} 2_{1}$ using the method of Coelho (2003). The structure was then solved by simulated annealing. The crystal structure of phase III could be re-solved to yield the same structure in repeated simulated annealing runs. The same solution was also obtained by the refinement of phase II coordinates: the difference between the phases largely lies in the orientation of the hydroxyl group and the two structures are similar enough that one can be refined into the other. During refinement the serine molecules were treated as variable metric rigid groups using the $Z$-matrix formalism available in TOPAS.
Lead (included in the sample as a pressure marker), tungsten carbide and nickel were also included in the refinement models. The weak tungsten carbide and nickel peaks both arise from the $\mathrm{P}-\mathrm{E}$ cell anvil cores which consist of cemented WC with a Ni binder. The refined cell dimensions of lead were used to calculate the applied pressure using a Birch-Murnaghan equation-of-state (Birch, 1947) using $V_{o}=30.3128 \AA^{3}$, $B_{o}=41.92 \mathrm{GPa}, B^{\prime}=5.72$. These parameters were derived by Fortes (2004) as averages of the values in Kuznetsov et al. (2002), Miller \& Schuele (1969) and Waldorf \& Alers (1962).

All intramolecular bond distances and angles were constrained to their ambient pressure values for all but the hydroxyl group, whose $\mathrm{O}-\mathrm{D}$ distance and $\mathrm{C}-\mathrm{O}-\mathrm{D}$ angle were refined. All torsion angles were also refined. All N-D and $\mathrm{C}-\mathrm{D}$ distances were constrained to 1.009 and $1.083 \AA$, respectively. All non-H atoms were refined with a common isotropic displacement parameter; the displacement parameters of the $\mathrm{D}$ atoms were equal to 1.5 times this value. The final Rietveld fits are shown in Fig. 1. Unit-cell dimensions and refinement parameters for L-serine-I at $4.6 \mathrm{GPa}$, L-serine-II at 5.2 and $7.3 \mathrm{GPa}$, and L-serine-III at $8.1 \mathrm{GPa}$ are included in Table 1. ${ }^{\mathbf{1}}$ Fourier difference maps calculated after the removal

\footnotetext{
${ }^{1}$ Supplementary data for this paper are available from the IUCr electronic archives (Reference: BS5027). Services for accessing these data are described at the back of the journal.
} 
Table 1

Experimental details.

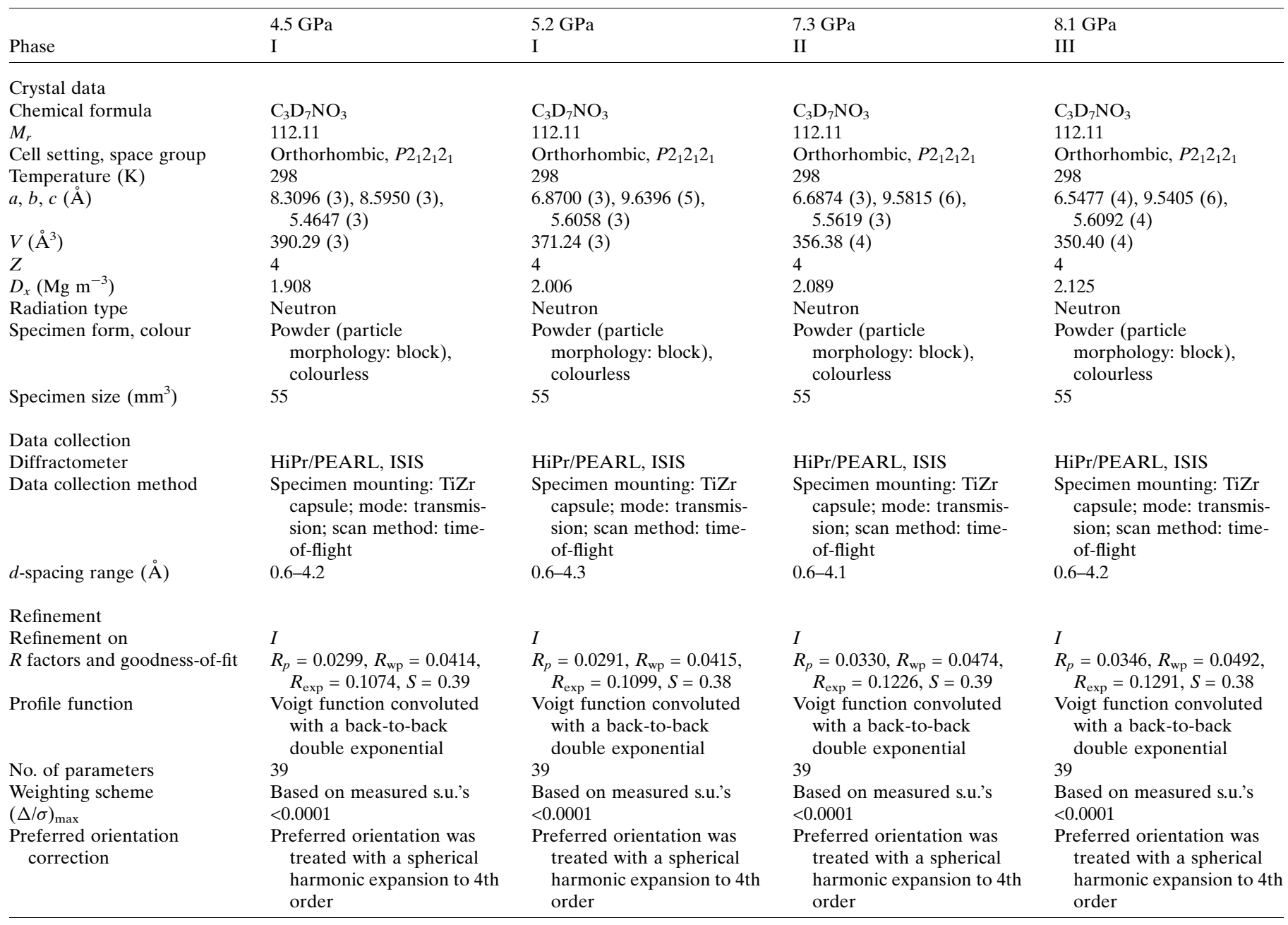

of some atoms returned the positions of the same atoms again; the success of this test attests to the quality of the data measured.

Crystal structures were visualized using the programs MERCURY (Bruno et al., 2002) and DIAMOND (Crystal Impact, 2004). Analyses were carried out using PLATON (Spek, 2003), as incorporated in the WIN-GX suite (Farrugia, 1999). Hirshfeld surface analysis was performed using the program CrystalExplorer (Wolff et al., 2005).

\section{Results and discussion}

\subsection{The response of L-serine-I- $d_{7}$ to $4.5 \mathrm{GPa}$}

We have previously investigated the response of L-serine-I to pressure using single-crystal X-ray diffraction (Moggach, Allan, Morrison, Parsons \& Sawyer, 2005). Boldyreva et al. (2005) also studied L-serine-I to $4.4 \mathrm{GPa}$, obtaining similar results. While deuterated materials can sometimes display different phase behaviour to their perhydrogenated analogues, the response of deuterated L-serine- $d_{7}$-I is essentially the same as that of L-serine- $h_{7}$ I up to $4.6 \mathrm{GPa}$. On increasing pressure to $5.2 \mathrm{GPa}$, the sample underwent a phase transition to $\mathrm{L}$-serine-II. The phase transition from L-serine-I to L-serineII is marked by a sudden decrease in volume (Fig. 2).

\subsection{Description of phases I and II}

We have discussed the structures of L-serine-I and II in detail in Moggach, Allan, Morrison, Parsons \& Sawyer (2005), and our motive for including a summary here is to highlight hydrogen-bonded motifs relevant to the development of Lserine-III, described below.

$C(5)$ chains are formed in both L-serine-I and II along $\mathbf{c}$ by N1D5...O2 hydrogen bonds (Figs. $3 a$ and $b$ ). A second $C(5)$ chain, generated from the first via a $2_{1}$ operation, is linked to the first through N1D6...O1 hydrogen bonds, and together the N1D5...O2 and N1D6...O 1 interactions form ribbons containing successive $R_{3}^{3}(11)$ ring motifs. The $\mathrm{CD}_{2} \mathrm{OD}$ side chains are distributed along the outside edges of these ribbons. In L-serine-I these link the ribbons into a layer via OD...OD...OD interactions (Fig. 3a). In L-serine-II the ribbons are connected into a layer by O3D $7 \cdots \mathrm{O} 2$ hydrogen bonds formed between the alcohol and carboxyl moieties (Fig. 
$3 b)$. The graph-sets thus-formed are $R_{3}^{3}(13)$ and $R_{3}^{2}(13)$, and the layers are stacked along the $a$ axis in both phases.

The layers are linked together in both phases I and II by N1D4 ...O2 interactions, which build another primary-level

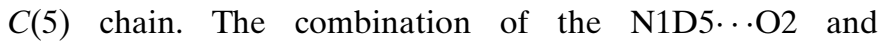
N1D4...O2 $C(5)$ chains along a and $\mathbf{c}$ builds a third set of secondary-level ring motifs, those having the descriptor $R_{4}^{3}(14)$ (Figs. $4 a$ and $b$ ). In fact, these chains can be viewed as building up another layer, which is parallel to the $a c$ plane. Overall, the structure consists of two sets of layers: one stacks along $\mathbf{a}$ and contains $R_{3}^{3}(11)$ and $R_{3}^{3}(13)$ ring motifs, the other stacks along b and contains $R_{4}^{3}(14)$ rings. In Moggach, Allan, Morrison, Parsons \& Sawyer (2005) we referred to these as the $A$ and $B$ layers, respectively.

\subsection{Comparison of L-serine-I at 4.5 GPa and L-serine-II at 5.2 GPa}

One difference between L-serine-I and -II is the position of the hydroxyl $\mathrm{H}$ atom. In our original X-ray study the position of this $\mathrm{H}$ atom was rather imprecisely determined, but our conclusions are confirmed by the present neutron study. The $\mathrm{O} \cdots \mathrm{O}$ and $\mathrm{D} \cdots \mathrm{O}$ distances in the O3D7... 2 2 hydroxylcarboxyl hydrogen bonds in phase II are substantially shorter than the O3D7...O3 hydroxyl-hydroxyl hydrogen bonds in phase I (Table 2).

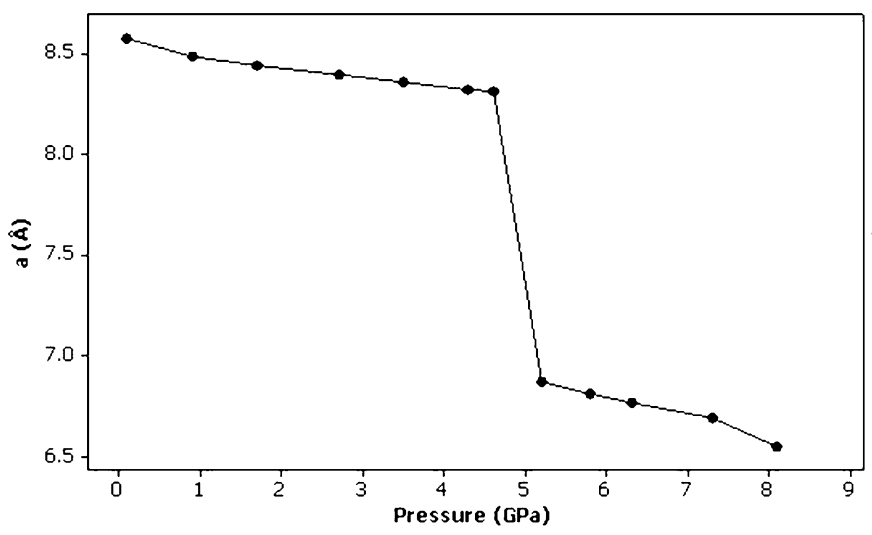

(a)

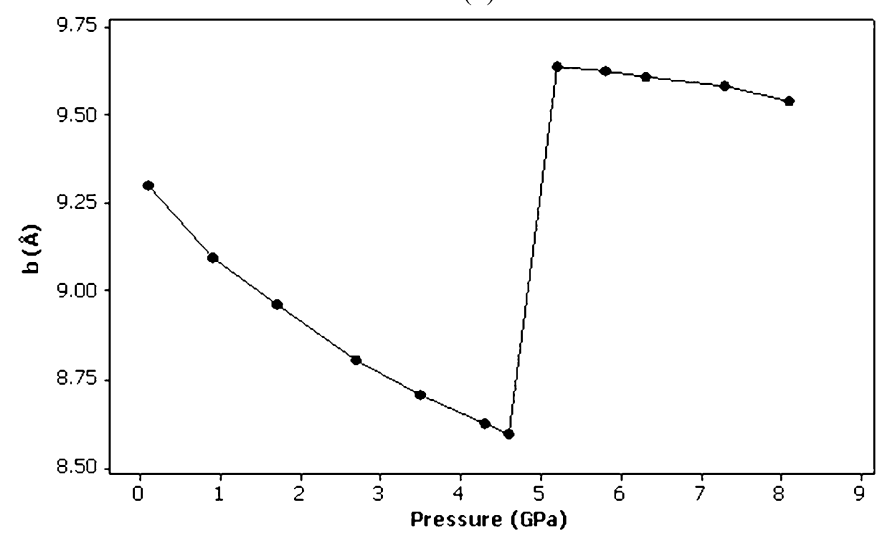

(b)
The conformations of the $R_{4}^{3}(14)$ rings in L-serine-I and -II represent a second difference between the crystal structures of the two phases. Voids observed in L-serine-I- $d_{7}$ at $4.6 \mathrm{GPa}$ at the centres of the $R_{4}^{3}(14)$ ring motifs are closed-up on undergoing the transition (Figs. $5 a$ and $b$ ). This is accomplished by:

(i) a displacement along the $+\mathbf{c}$ and $-\mathbf{c}$ directions by successive $C(5)$ chains formed by N1D5...O2 hydrogen bonds, and

(ii) a shortening of the distance between the chains along the a direction (Figs. $6 a$ and $b$ ).

The opposite directions of displacement of the $C(5)$ chains break the O3D7...O3 hydroxyl-hydroxyl hydrogen bonds which are replaced by $\mathrm{O} 3 \mathrm{D} 7 \cdots \mathrm{O} 2$ hydroxyl-carboxylate hydrogen bonds in phase II.

CD $\cdots O$ o contacts are also more numerous in L-serine-II than in L-serine-I. All $\mathrm{C}-\mathrm{H}$ bonds act as donors in two hydrogen-bonding interactions which span the $R_{3}^{2}(13)$ and $R_{4}^{3}(14)$ rings (Table 2, Figs. $3 b$ and $4 \mathrm{~b}$ ).

\subsection{L-Serine-II between 5.2 and $7.3 \mathrm{GPa}$}

As the pressure on L-serine-II is increased from 5.2 to $7.3 \mathrm{GPa}$, the largest compression occurs along the a direction, with the $a$ axis decreasing in length by $2.7 \%$; the $b$ and $c$ axes reduce by only 0.6 and $0.8 \%$, respectively. The relative displacement of the N1D5 ...O2 C(5) chains and the reduction in the distance between the chains both continue up to

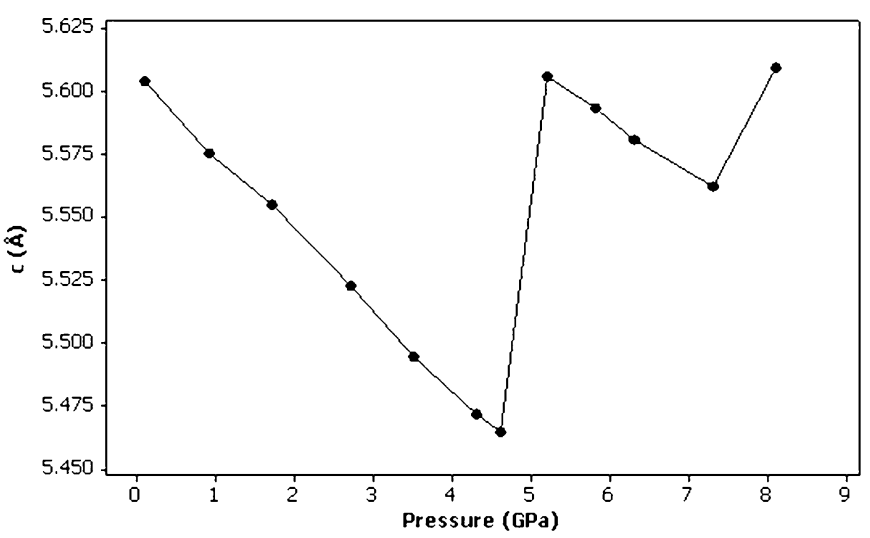

(c)

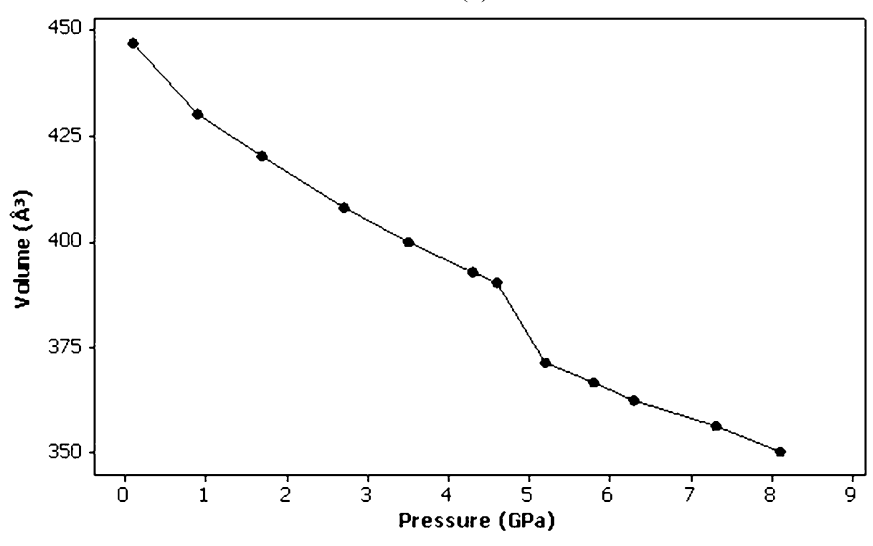

(d)

Figure 2

Variation of the lattice parameters $(a)-(c)$ and volume $(d)$ of L-serine as a function of pressure (GPa). 

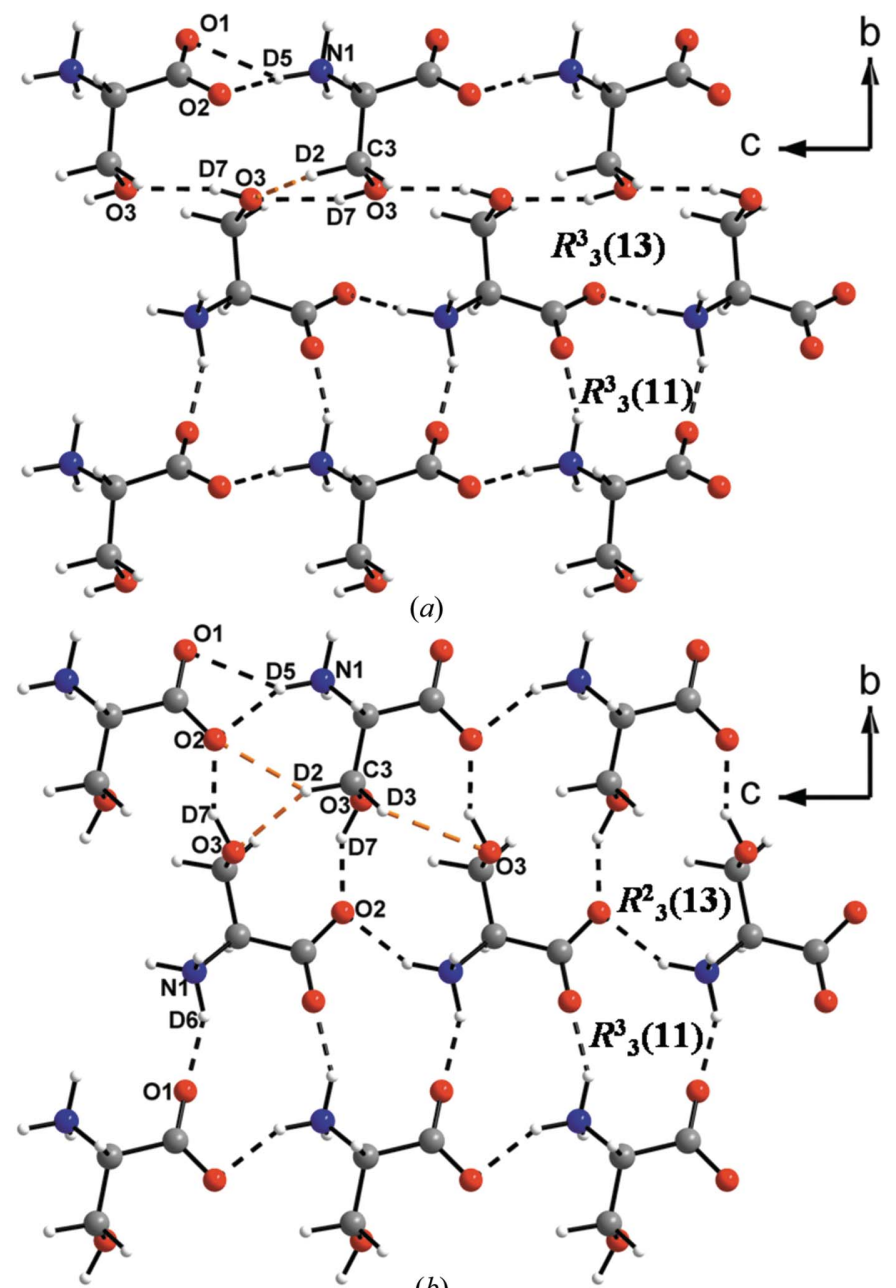

(b)

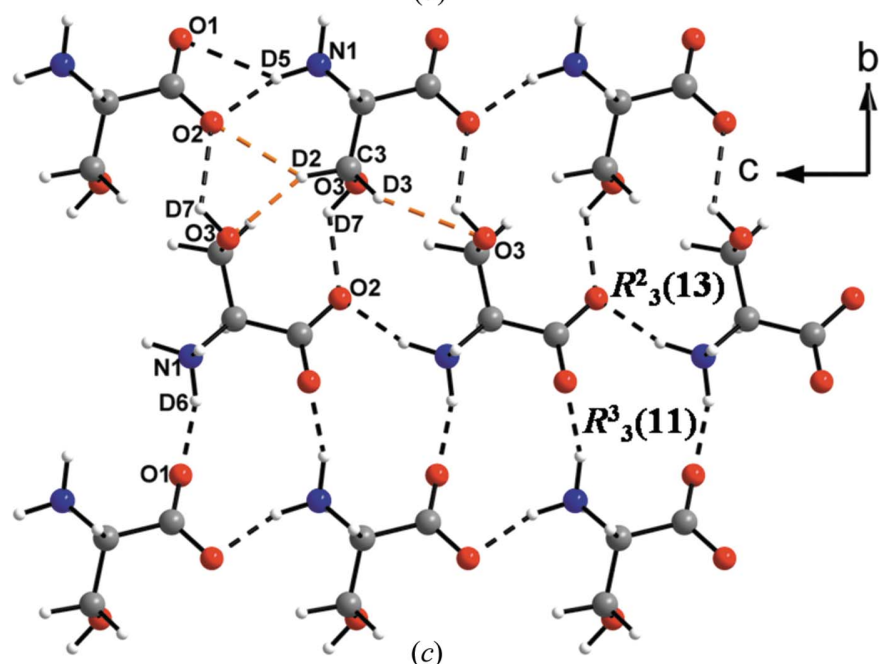

(c)

Figure 3

(a) L-Serine-I at 4.6 GPa, $(b)$ L-serine-II at $5.2 \mathrm{GPa}$ and $(c)$ L-serine-III at $8.1 \mathrm{GPa}$ as viewed along a. $R_{3}^{3}(13), R_{3}^{2}(13)$ and $R_{3}^{3}(11)$ ring motifs are shown. ND . . O and OD ... O hydrogen bonds are drawn as black dotted lines, while $\mathrm{CD} \cdots \mathrm{O}$ interactions are drawn as orange dotted lines. For clarity, the bifurcated interaction $\mathrm{N} 1 \mathrm{D} 5 \cdots \mathrm{O} 1 / \mathrm{O} 2$ and $\mathrm{CD} \cdots \mathrm{O}$ interactions are shown in the top-left corner only. These layers are referred to as the $A$ layers in the text. Colour scheme: $\mathrm{C}$ grey, $\mathrm{H}$ white, $\mathrm{N}$ blue and $\mathrm{O}$ red.
7.3 GPa, leading to further compression of the voids within $R_{4}^{3}(14)$ ring motifs (Figs. $6 a$ and $b$, and $5 c$ ).

In the most compressible ND...O hydrogen bond, N1D5 ...O 1, the D...O distance decreases by 0.075 (11) $\AA$ between 5.2 and $7.3 \mathrm{GPa}$. This hydrogen bond is the longest of its type at $5.2 \mathrm{GPa}$, and its shortening increases the bifurcation in the interaction N1D5 . .OO1/O2. N1D6 . .O 1, which is short anyway at $5.2 \mathrm{GPa}$, remains relatively unchanged. The largest

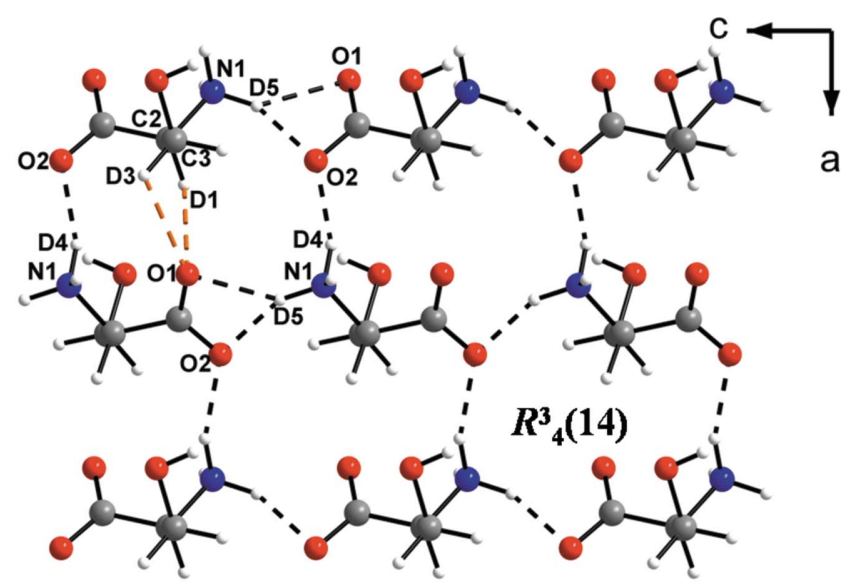

(a)

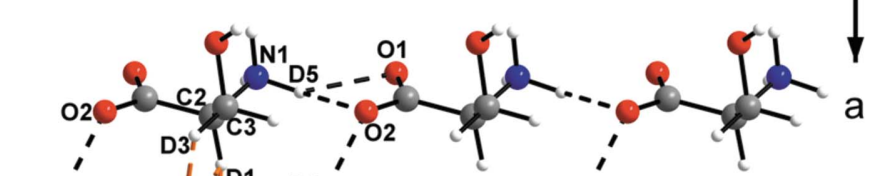

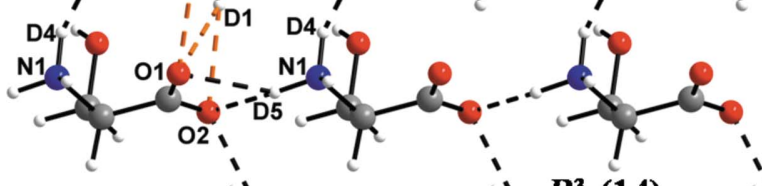

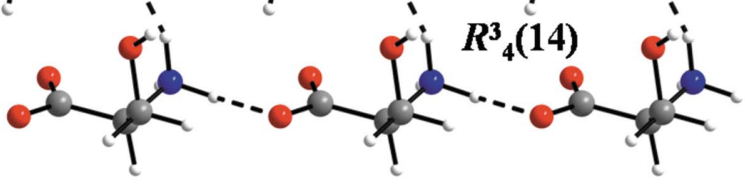

(b)
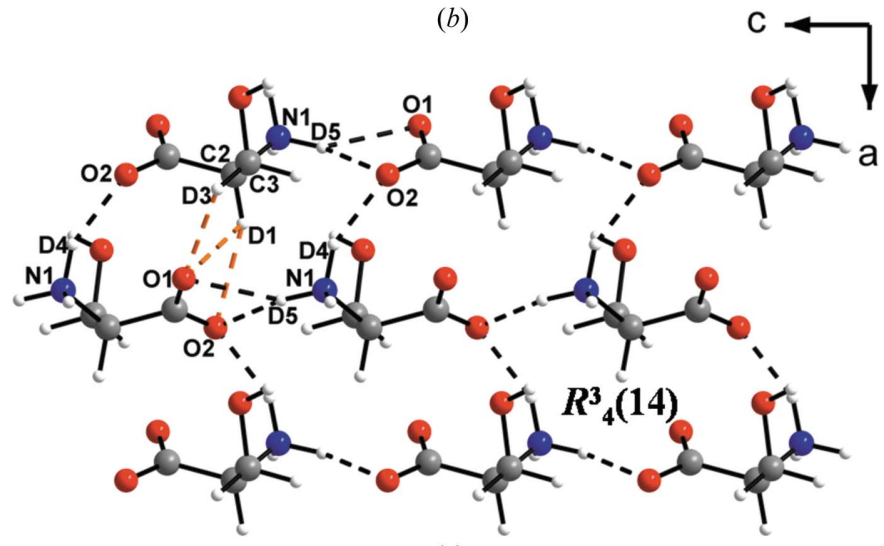

(c)

Figure 4

(a) L-Serine-I at $4.6 \mathrm{GPa},(b) \mathrm{L}$-serine-II at $5.2 \mathrm{GPa}$ and (c) L-serine-III at $8.1 \mathrm{GPa}$ as viewed along b. $R_{4}^{3}(14)$ ring motifs are shown. ND $\cdots \mathrm{O}$ and $\mathrm{OD} \cdots \mathrm{O}$ hydrogen bonds are drawn as black dotted lines, while $\mathrm{CD} \cdots \mathrm{O}$ interactions are drawn as orange dotted lines. For clarity, the bifurcated interaction N1D5 $\cdots \mathrm{O} 1 / \mathrm{O} 2$ and $\mathrm{CD} \cdots \mathrm{O}$ interactions are shown in the top-left corner only. These layers are referred to as the $B$ layers in the text. The colour scheme is the same as in Fig. 3. 
Table 2

Hydrogen-bonding parameters $\left(\AA,^{\circ}\right)$ in L-serine.

Roman numerals refer to the phase of L-serine.

\begin{tabular}{|c|c|c|c|c|}
\hline Pressure (GPa) & $4.6^{I}$ & $5.2^{\mathrm{II}}$ & $7.3^{\mathrm{II}}$ & $8.1^{\mathrm{III}}$ \\
\hline \multicolumn{5}{|l|}{$\mathrm{N} 1 \mathrm{D} 4 \cdots \mathrm{O} 2^{\mathrm{i}}$} \\
\hline $\mathrm{N} 1 \cdots \mathrm{O} 2$ & $2.743(11)$ & $2.821(17)$ & $2.764(18)$ & $2.836(15)$ \\
\hline $\mathrm{D} 4 \cdots \mathrm{O} 2$ & $1.839(11)$ & 1.877 (19) & $1.85(2)$ & $1.890(16)$ \\
\hline$\angle \mathrm{N} 1 \mathrm{D} 4 \mathrm{O} 2$ & $147.3(5)$ & $154.3(12)$ & $149.3(12)$ & $154.8(7)$ \\
\hline \multicolumn{5}{|l|}{$\mathrm{N} 1 \mathrm{D} 5 \cdots \mathrm{O} 1^{\mathrm{ii}}$} \\
\hline $\mathrm{N} 1 \cdots \mathrm{O} 1$ & $3.012(5)$ & $3.071(4)$ & 3.008 (4) & 3.085 (3) \\
\hline 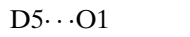 & $2.215(8)$ & $2.243(8)$ & $2.168(8)$ & $2.304(7)$ \\
\hline$\angle \mathrm{N} 1 \mathrm{D} 5 \ldots \mathrm{O} 1$ & $134.8(5)$ & $138.4(5)$ & $139.6(5)$ & $133.3(4)$ \\
\hline \multicolumn{5}{|l|}{$\mathrm{N} 1 \mathrm{D} 5 \cdots \mathrm{O} 2^{\mathrm{ii}}$} \\
\hline $\mathrm{N} 1 \cdots \mathrm{O} 2$ & $2.714(10)$ & $2.823(10)$ & $2.829(11)$ & $2.776(9)$ \\
\hline $\mathrm{D} 5 \cdots \mathrm{O} 2$ & $1.762(13)$ & 1.875 (13) & $1.902(15)$ & $1.796(11)$ \\
\hline$\angle \mathrm{N} 1 \mathrm{D} 5 \mathrm{O} 2$ & $155.7(5)$ & $155.2(9)$ & $151.3(10)$ & $162.9(4)$ \\
\hline \multicolumn{5}{|l|}{$\mathrm{N} 1 \mathrm{D} 6 \cdots \mathrm{O} 1^{\mathrm{iii}}$} \\
\hline $\mathrm{N} 1 \cdots \mathrm{O} 1$ & $2.631(14)$ & $2.642(15)$ & $2.640(17)$ & $2.694(18)$ \\
\hline D6...O1 & $1.683(14)$ & 1.733 (14) & $1.735(16)$ & $1.710(18)$ \\
\hline$\angle \mathrm{N} 1 \mathrm{D} 6 \mathrm{O} 1$ & $154.6(5)$ & $147.8(6)$ & $147.1(7)$ & $164.2(8)$ \\
\hline \multicolumn{5}{|l|}{$\mathrm{O} 3 \mathrm{D} 7 \cdots \mathrm{O} 3^{\mathrm{iv}}$} \\
\hline $\mathrm{O} 3 \ldots \mathrm{O} 3$ & $2.780(5)$ & - & - & - \\
\hline $\mathrm{D} 7 \ldots \mathrm{O} 3$ & $1.954(18)$ & - & - & - \\
\hline$\angle \mathrm{O} 3 \mathrm{D} 7 \mathrm{O} 3$ & $156(2)$ & - & - & - \\
\hline \multicolumn{5}{|l|}{$\mathrm{O} 3 \mathrm{D} 7 \cdots \mathrm{O} 2^{\mathrm{iv}}$} \\
\hline $\mathrm{O} 3 \cdots \mathrm{O} 2$ & - & $2.576(16)$ & $2.516(17)$ & $2.638(15)$ \\
\hline $\mathrm{D} 7 \cdots \mathrm{O} 2$ & - & 1.67 (3) & $1.56(3)$ & $2.05(3)$ \\
\hline$<\mathrm{O} 3 \mathrm{D} 7 \mathrm{O} 2$ & - & $168(3)$ & $174(3)$ & $122(2)$ \\
\hline \multicolumn{5}{|l|}{$\mathrm{O} 3 \mathrm{D} 7 \cdots \mathrm{O} 1^{\mathrm{v}}$} \\
\hline $\mathrm{O} 3 \cdots \mathrm{O} 1$ & - & - & - & $2.775(13)$ \\
\hline $\mathrm{D} 7 \ldots \mathrm{O} 1$ & & & & $1.95(3)$ \\
\hline$\angle \mathrm{O} 3 \mathrm{D} 7 \mathrm{O} 1$ & & & & $152(2)$ \\
\hline \multicolumn{5}{|l|}{$\mathrm{C} 2 \mathrm{D} 1 \cdots \mathrm{O} 1^{\mathrm{vi}}$} \\
\hline $\mathrm{D} 1 \cdots \mathrm{O} 1$ & $2.499(14)$ & $2.438(16)$ & $2.350(17)$ & $2.341(16)$ \\
\hline$\angle \mathrm{C} 2 \mathrm{D} 1 \mathrm{O} 1$ & $119.2(4)$ & $111.1(4)$ & 109.7 (4) & $105.9(4)$ \\
\hline \multicolumn{5}{|l|}{$\mathrm{C} 2 \mathrm{D} 1 \cdots \mathrm{O} 2^{\mathrm{vi}}$} \\
\hline $\mathrm{D} 1 \cdots \mathrm{O} 2$ & - & $2.385(15)$ & $2.338(16)$ & $2.322(14)$ \\
\hline$\angle \mathrm{C} 2 \mathrm{D} 1 \mathrm{O} 2$ & & $159.6(4)$ & $157.9(4)$ & $154.7(4)$ \\
\hline \multicolumn{5}{|l|}{$\mathrm{C} 3 \mathrm{D} 2 \cdots \mathrm{O} 2^{\mathrm{ii}}$} \\
\hline $\mathrm{D} 2 \cdots \mathrm{O} 2$ & - & $2.389(12)$ & $2.297(12)$ & $2.378(11)$ \\
\hline$\angle \mathrm{C} 3 \mathrm{D} 2 \mathrm{O} 2$ & - & $128.2(7)$ & $129.7(8)$ & $129.2(7)$ \\
\hline \multicolumn{5}{|l|}{$\mathrm{C} 3 \mathrm{D} 2 \cdots \mathrm{O} 3^{\mathrm{iv}}$} \\
\hline $\mathrm{D} 2 \cdots \mathrm{O} 3$ & $2.41(3)$ & $2.44(3)$ & $2.39(3)$ & $2.32(2)$ \\
\hline$\angle \mathrm{C} 3 \mathrm{D} 2 \mathrm{O} 3$ & $114.5(5)$ & $131.3(8)$ & $132.5(9)$ & $152.7(7)$ \\
\hline \multicolumn{5}{|l|}{$\mathrm{C} 3 \mathrm{D} 3 \cdots \mathrm{O} 3^{\mathrm{vii}}$} \\
\hline $\mathrm{D} 3 \cdots \mathrm{O} 3$ & - & $2.37(3)$ & $2.32(2)$ & $2.26(2)$ \\
\hline$\angle \mathrm{C} 3 \mathrm{D} 3 \mathrm{O} 3$ & - & $132.9(10)$ & $134.1(11)$ & $157.2(12)$ \\
\hline \multicolumn{5}{|l|}{$\mathrm{C} 3 \mathrm{D} 3 \cdots \mathrm{O} 1^{\mathrm{vi}}$} \\
\hline D3...O1 & $2.420(15)$ & $2.36(2)$ & $2.32(2)$ & $2.229(17)$ \\
\hline$\angle \mathrm{C} 3 \mathrm{D} 3 \mathrm{O} 1$ & $105.7(6)$ & $109.5(7)$ & $109.5(7)$ & $113.4(6)$ \\
\hline
\end{tabular}

Symmetry codes: (i) $-\frac{1}{2}+x, \frac{3}{2}-y, 1-z$; (ii) $x, y, 1+z$; (iii) $\frac{3}{2}-x, 2-y, \frac{1}{2}+z$; (iv) $\frac{3}{2}-x, 1-y, \frac{1}{2}+z ; \quad$ (v) $\quad 1-x,-\frac{1}{2}+y, \frac{3}{2}-z ; \quad$ (vi) $\quad \frac{1}{2}+x, \frac{3}{2}-y, 1-z ; \quad$ (vii) $\frac{3}{2}-x, 1-y,-\frac{1}{2}+z$.

change occurs for O3D7...O2, which was formed in the transition from phase I to phase II: by $7.3 \mathrm{GPa}$ the $\mathrm{O} \cdots \mathrm{O}$ distance in this contact measures only 2.516 (17) $\AA$, which is very short. Although it is short, O3D7...O2 is unsymmetrical, with $\mathrm{O} 3-\mathrm{D} 7$ and D7 ...O2 measuring 0.97 (2) and 1.56 (3) $\AA$, respectively.

The shortest $\mathrm{OH}$. . O hydrogen bond found under ambient pressure conditions [2.474 (2) $\AA$ ] occurs in the CSD refcode AMHACY (Leban, 1974). This, however, corresponds to a symmetrical hydrogen bond where the $\mathrm{H}$ atom is shared equally between adjacent carboxyl groups from neighbouring molecules. Strong hydrogen bonds of this type are common when formed between acids and complementary bases
(Steiner, 2002). If this search is limited to hydrogen-bonding interactions between a free hydroxyl group donor (bonded to an alkyl $\mathrm{CH}_{2}$ ) and carboxyl group acceptor, the shortest interaction observed under ambient conditions measures 2.584 (2) $\AA$ for GOLWIN (Tusvik et al., 1999). The $\mathrm{O} 3 \mathrm{D} 7 \cdots \mathrm{O} 2$ hydrogen bond therefore appears to be pushed to below this lower limit observed under ambient pressure conditions, and on increasing pressure further the structure undergoes another phase transition to a second high-pressure phase, L-serine-III.

\subsection{Structure of L-serine-III at $8.1 \mathrm{GPa}$}

The phase transition from L-serine-II to L-serine-III, unlike that from phase I to II, occurs with only a modest decrease in volume (Fig. $2 d$ ); the volume per non-H atom at $8.1 \mathrm{GPa}$ is only $12.5 \AA^{3}$. Overall the structure is closely related to the structures of phases I and II (Figs. $3 c$ and $4 c$ ), and the structure can again be considered in terms of layers which stack along the $\mathbf{a}$ and $\mathbf{b}$ directions.

The $R_{4}^{3}(14)$ ring motifs in the $B$ layers appear longer and thinner than they do in L-serine-I and -II (Figs. $4 b$ and $c$ ), and over the course of the II-to-III phase transition the relative displacement of neighbouring N1D5 ..O $2 C(5)$ chains in the $+\mathbf{c}$ and $-\mathbf{c}$ directions continues (Fig. $6 a$ ). The associated compression along the $a$ direction also continues (Fig. 6b) and at $8.1 \mathrm{GPa}$ there are no visible voids within the $R_{4}^{3}(14)$ rings (Fig. 5d). Small voids present at the centre of $R_{3}^{2}(13)$ ring motifs of the $A$ layers remain essentially unchanged through the transition, while no observable voids can be observed at the centres of $R_{3}^{3}(11)$ ring motifs in phase II or III.

The phase transition from L-serine-I to L-serine-II was, in part, characterized by a change in the hydrogen bonds formed by the hydroxyl group, O3D7. A further change in hydrogen bonding in this region of the structure also occurs in the transition from phase II to phase III. In phase II the hydrogen bonding from O3D7 was confined to one $A$ layer, but in phase III the hydrogen bonding from D7 becomes bifurcated between the same O3D7 . O 2 interaction that was present in phase II, and a new O3D7...O1 hydrogen bond which connects different $A$ layers together. This change in the hydrogen-bonding environment of the O3D7 group may explain the findings of Kolesnik et al. (2005), who observed that the largest changes in the Raman spectra of L-serine on undergoing the transition from phase II to III occurred in bands at 1250 (HOC bend) and $1430 \mathrm{~cm}^{-1}$ ( $\mathrm{CO}_{2}$ sym. str). When viewed in projection the $A$ and $B$ layers seem very similar in phases II and III: the difference lies in the way they are connected together and this is clearer in the $c$-axis projection illustrated in Fig. 7.

The O3...O2 distance within the O3D7...O2 hydrogen bonds in phase II is very short [2.516 (17) $\AA$ ] at $7.3 \mathrm{GPa}$ and the structure presumably experiences significant strain in this region. It seems reasonable to suggest that the II-to-III transition is driven by the need to alleviate close contacts about $\mathrm{O} 3$ in L-serine-II as pressure is increased beyond $7.3 \mathrm{GPa}$. 


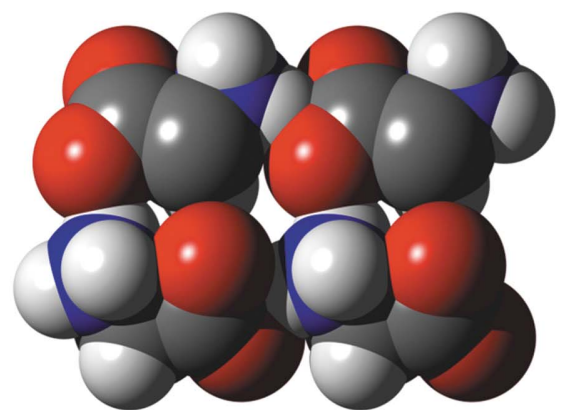

(a)

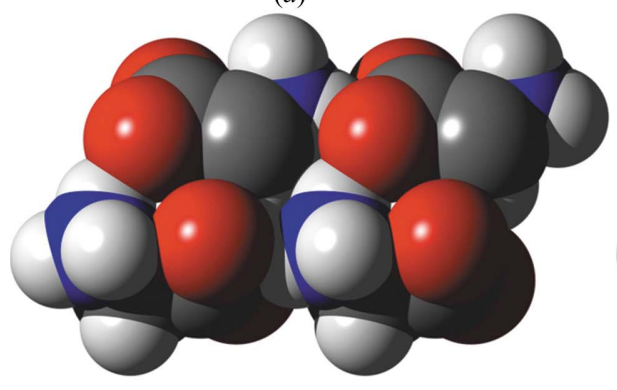

(c)

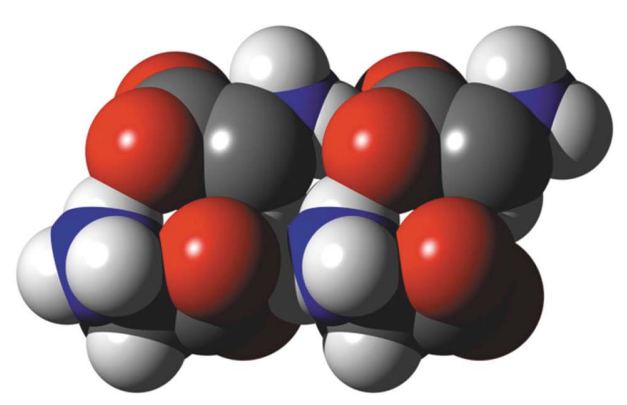

(b)

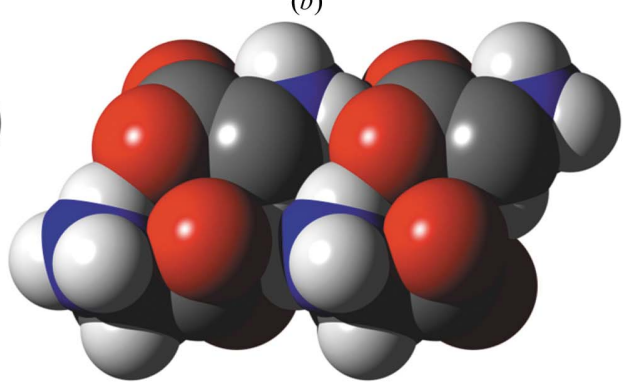

(d)

Figure 5

Space-filling plots for $R_{4}^{3}(14)$ ring motifs in $(a)$ L-serine-I at $4.6 \mathrm{GPa},(b)$ L-serine-II at $5.2 \mathrm{GPa},(c)$ Lserine-II at $7.3 \mathrm{GPa}$ and $(d)$ L-serine-III at $8.1 \mathrm{GPa}$ as viewed along b. The $\mathrm{CD}_{2} \mathrm{OD}$ side groups have been removed for clarity. The colour scheme is the same as in Fig. 3.

On increasing the pressure in $\mathrm{L}$ serine-I to between 0.1 to $4.6 \mathrm{GPa}$, the drive to compress the hydrogenbonding interactions is represented clearly on the fingerprint plots with $d_{e}$ values driven to shorter distances (Figs. $8 a$ and $b$ ). In particular, the two long spikes present in all fingerprint plots represent ND... and OD...O hydrogen-bonding interactions, which can clearly be observed moving toward smaller values of $d_{e}$ in L-serine-I (Figs. $8 a$ and $b)$. The number of points at larger values of $d_{e}$ and $d_{i}$ also decrease on increasing pressure, giving a more compact plot at 4.6 GPa; the fingerprint plot is still more compact at $5.2 \mathrm{GPa}$ (Fig. $8 c$ ). This illustrates the compression of voids within $R_{3}^{3}(11)$ and $R_{4}^{3}(14)$ ring motifs within $A$ and $B$ layers, respectively. A 'hump' appears between the spikes at $4.5 \mathrm{GPa}$, becoming more prominent at
In general, at elevated pressures the influence of hydrogen bonds as structure-directing interactions is expected to diminish relative to the need to pack molecules more efficiently and several of the $\mathrm{ND} \cdots \mathrm{O}$ and OD..O hydrogen bonds in phase III at $8.1 \mathrm{GPa}$ are longer than those in phase II at $7.3 \mathrm{GPa}$. In L-serine-II the hydrogen-bonding capacity of the $\mathrm{C}-\mathrm{D}$ bonds is saturated and so the transition from phase II to III does not result in an increase in number of CD..O interactions, with the six interactions present in L-serine-II, still observed in L-serine-III.

\subsection{Changes in CD . O and D ...D interactions: Hirshfeld fingerprint plot analysis}

McKinnon et al. (2004) have recently described how a Hirshfeld surface analysis can assist in the interpretation of differences between related crystal structures. Hirshfeld surfaces are created by partitioning space within a crystal structure into regions where the electron density from a sum of spherical atoms (the promolecule) dominates over the sum of the electron density of the crystal (the procrystal). The surfaces surround each molecule in a crystal structure, enabling various properties relating to packing to be calculated. Two such properties are $d_{e}$ (the distance from the surface to the nearest atom in another molecule) and $d_{i}$ (the distance from the surface to the nearest atom in the molecule itself). Plots of $d_{e}$ against $d_{i}$ are referred to as fingerprint plots and they encode information on the overall three-dimensional packing characteristics in a two-dimensional diagram. Fingerprint plots for the structures obtained in this study are shown in Fig. 8.

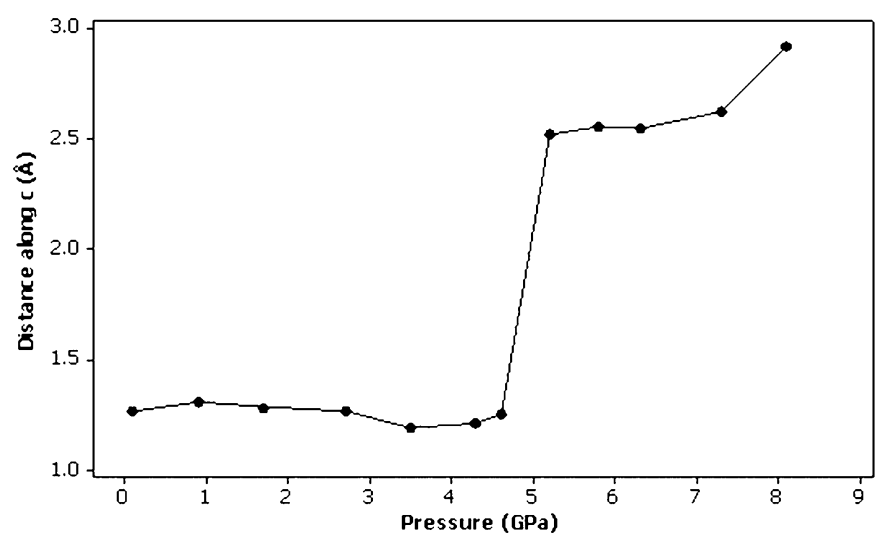

(a)

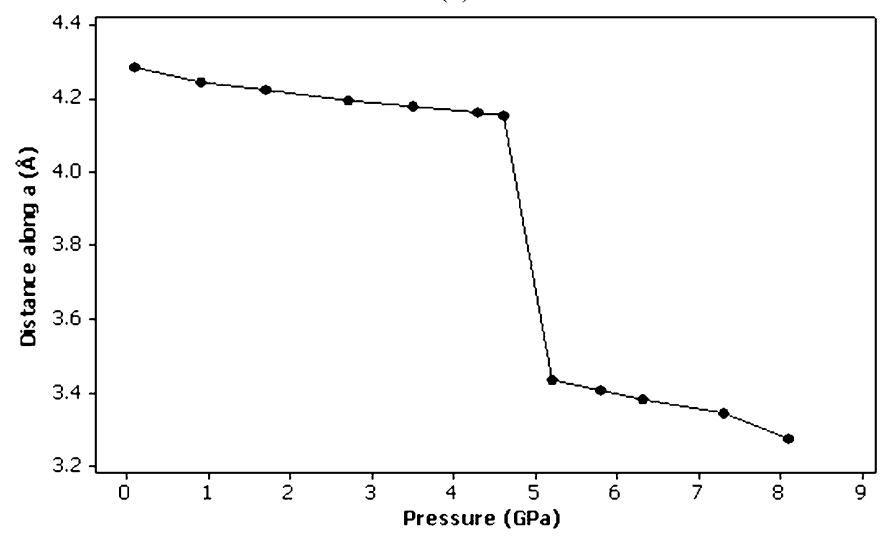

(b)

Figure 6

Graphs showing the distances between the centroids of the molecules in neighbouring $C(5)$ chains along $(a) \mathbf{c}$ and $(b) \mathbf{a}$. 


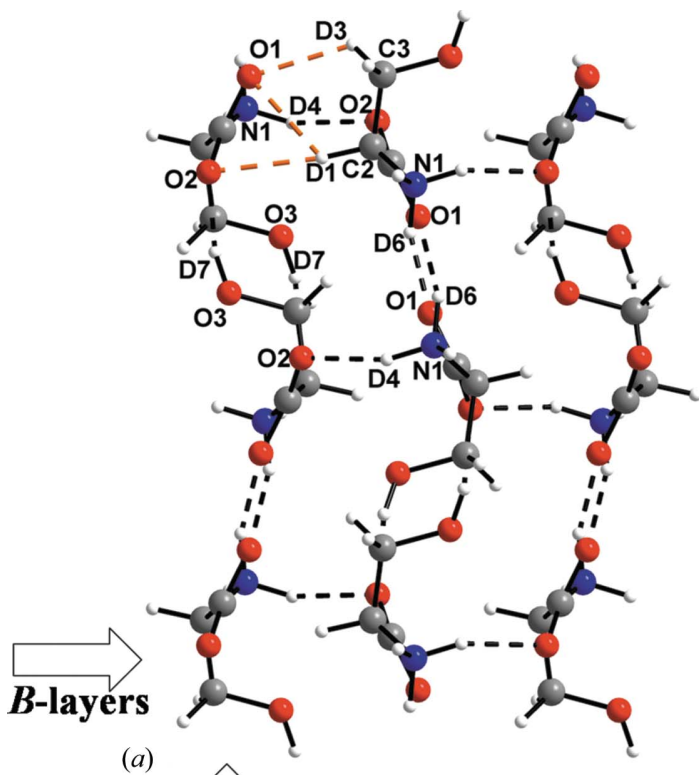

(a)

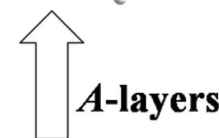

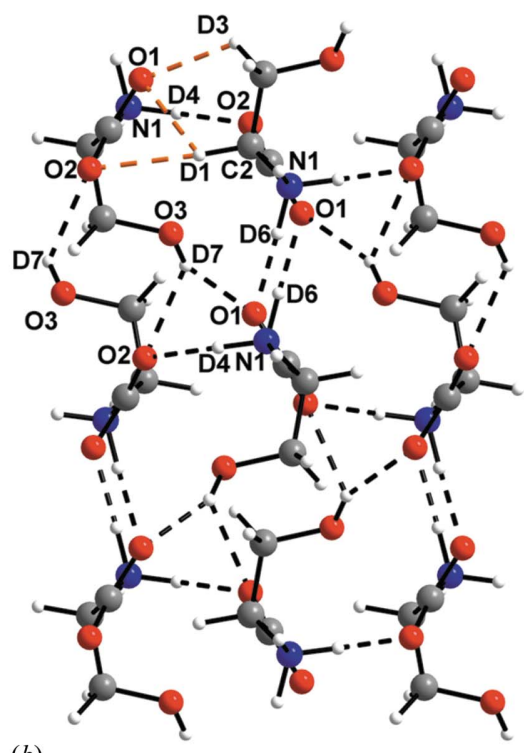

(b)

5.2 GPa. This corresponds to close D...D contacts. The shortest of these contacts at 4.6 GPa, D3...D6, forms between $A$ layers between D atoms from $\mathrm{CD}_{2}$ and ammonium groups of adjacent molecules, respectively. D...D contacts become more numerous at $5.2 \mathrm{GPa}$, for example a D3. .D6 distance formed along the $a$ direction decreases from 2.19 to $2.08 \AA$.

On increasing the pressure further to $7.3 \mathrm{GPa}$, the fingerprint plots look much the same, although a slight movement of the $\mathrm{NH} \cdots \mathrm{O} / \mathrm{OD} \cdots \mathrm{O}$ spikes toward the lower values of $d_{e}$ can be observed (Figs. $8 c$ and $d$ ). The 'hump' between the spikes at 7.3 GPa also appears to sharpen and move to larger values of $d_{e}$. This is accounted for by the movement of the hydroxyl OD group, which at $5.2 \mathrm{GPa}$ forms

Figure 7

(a) L-Serine-II at $5.2 \mathrm{GPa}$ and $(b)$ L-serine-III at $8.1 \mathrm{GPa}$ as viewed along $\mathbf{c} . A$ and $B$ layers as referred to in the text are labelled. ND ... O and OD ... O hydrogen bonds are drawn as black dotted lines, while CD ..O interactions are drawn as orange dotted lines. For clarity, CD . O interactions are shown in the top-left corner only. Note the formation of the O1D7 . . O1 interaction in L-serine-III at 8.1 GPa. The colour scheme is the same as in Fig. 3.
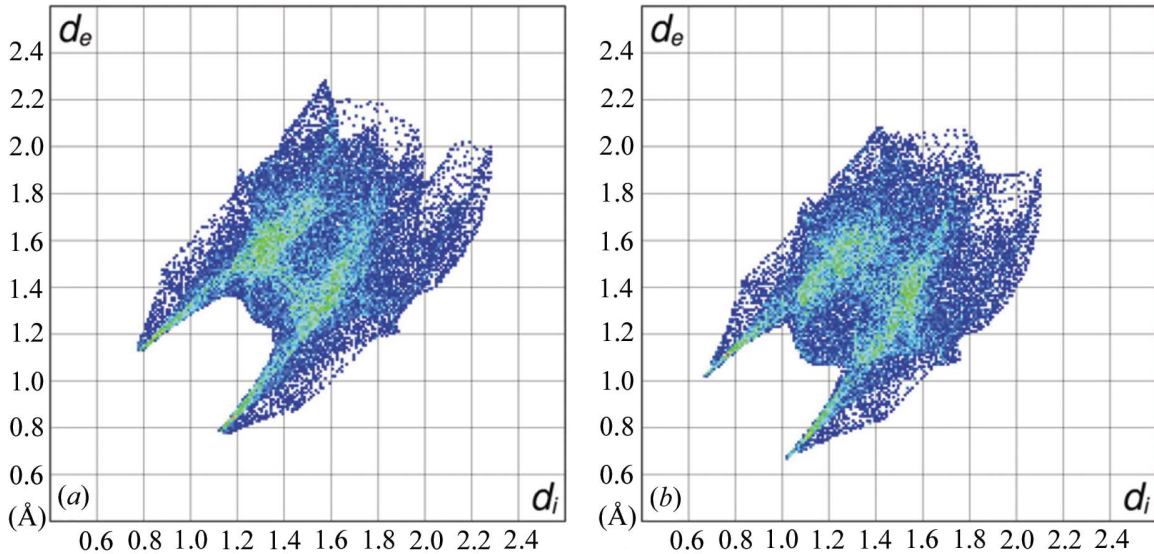
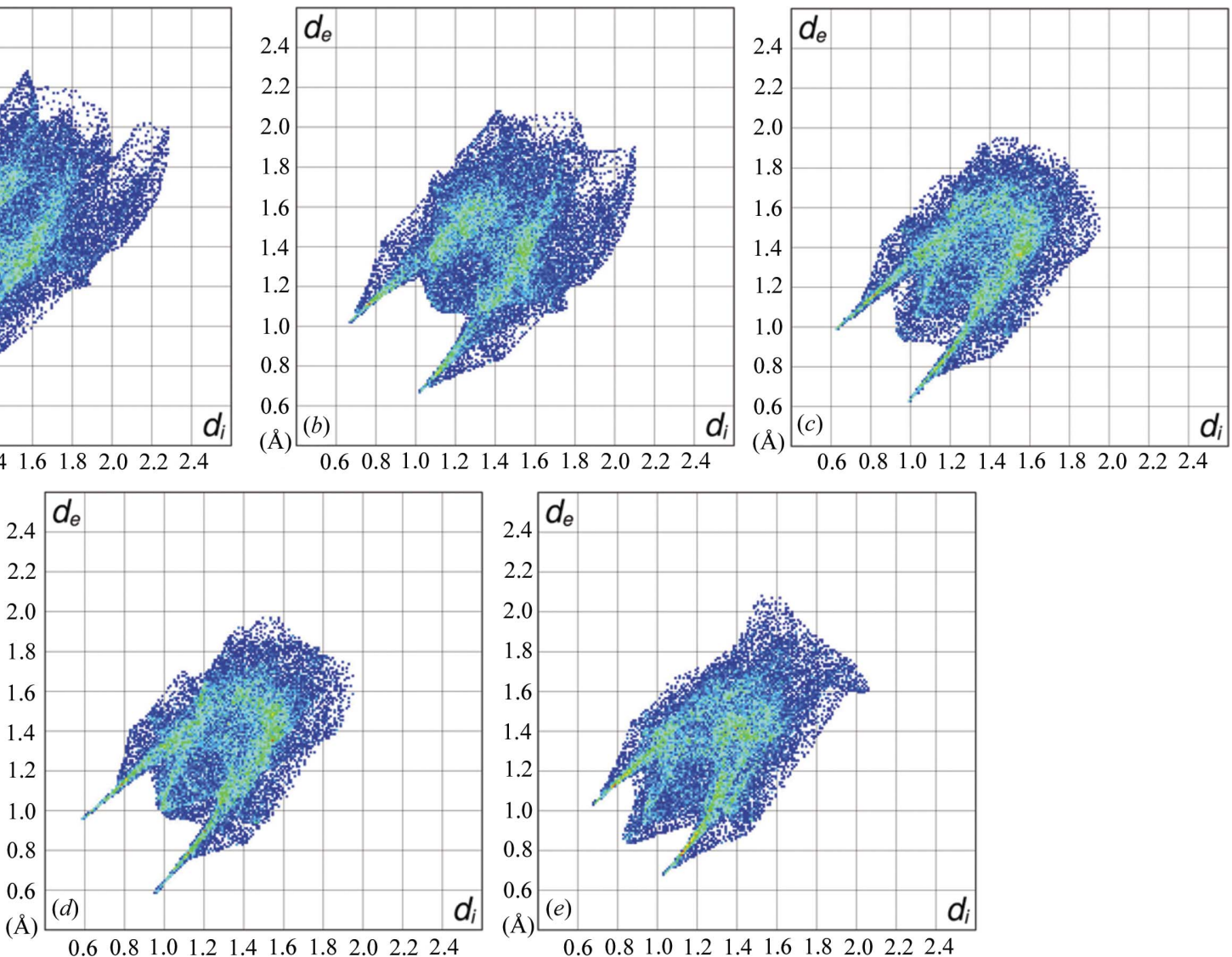

Figure 8

Two-dimensional fingerprint plots for $(a)$ L-serine-I at $0.1 \mathrm{GPa},(b)$ L-serine-I at 4.6 GPa, $(c)$ L-serine-II at 5.2 GPa, $(d)$ L-serine-II at $7.3 \mathrm{GPa}$ and $(e)$ Lserine-III at $8.1 \mathrm{GPa}$. 


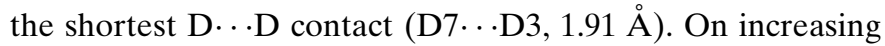
the pressure to $7.3 \mathrm{GPa}$, this close contact is actually relieved by the hydroxyl group, which twists, making the O3D7 ‥O2 hydrogen bond more linear, while increasing the D7...D3 distance $(1.98 \AA)$. This movement of the hydroxyl group can be quantified by comparing the $\mathrm{D} 7-\mathrm{O} 3-\mathrm{C} 3-\mathrm{C} 2$ torsion angle at $5.2 \mathrm{GPa}\left(-178.5^{\circ}\right)$ and $7.3 \mathrm{GPa}\left(+172.3^{\circ}\right)$. Nevertheless, on increasing pressure to $7.3 \mathrm{GPa}$, the number of close D $\cdots$ D contacts does increase, represented in Fig. 8(d) as an increase in the green colour within the 'hump' region of the plot.

At $8.1 \mathrm{GPa}$ the spikes corresponding to ND $\cdots$ O hydrogen bonds appear at larger values of $d_{e}$; this is caused by the increased lengths of ND $\cdots \mathrm{O}$ and $\mathrm{OD} \cdots \mathrm{O}$ contacts in phase III referred to above (Figs. $8 d$ and $e$ ). We have suggested above that the II-to-III transition is driven by the need to relieve short intermolecular contacts, and the similarity of the volumes of phases II and III suggests that the transition does not result in markedly more efficient packing. However, there is a pronounced increase in the number of close $\mathrm{D} \cdots \mathrm{D}$ contacts between the spikes in the fingerprint plot shown in Figs. $8(d)$ and $(e)$. Close contacts between $\mathrm{H}$ atoms are characteristic of the fingerprint plots obtained for alkanes (McKinnon et al., 2004) and in serine their gradually increasing prominence illustrates much more graphically than tables of data the tendency for efficient packing to compete with hydrogen bonding as structure-directing phenomena.

\section{Summary}

We have previously described a single-crystal X-ray study in which we observed a single crystal to single crystal phase transition from L-serine-I to an orthorhombic high-pressure phase, L-serine-II, between 4.8 and 5.4 GPa. The same transition has also been observed by neutron powder diffraction in perdeuterated L-serine between 4.6 and 5.2 GPa. The I-to-II transition is accompanied by a large reduction in volume, achieved by opposite displacements of successive $C(5)$ chains formed by ND ...O hydrogen bonds and a shortening of the distance between the chains. Overall, this closes up the voids present in hydrogen-bonded ring motifs, enabling the reduction in volume to occur. The opposite direction of the

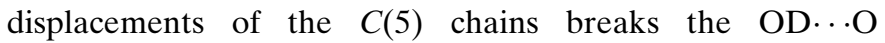
hydroxyl-hydroxyl hydrogen bonds present in phase I. These are replaced by OD ...O hydroxyl-carboxylate interactions in phase II. The change in position of the hydroxyl $\mathrm{H}$ atom was clearly demonstrated in this study.

Compression of L-serine-II from 5.2 to $7.3 \mathrm{GPa}$ caused the $\mathrm{O} \cdots \mathrm{O}$ distance in the newly formed OD $\cdots \mathrm{O}$ hydroxylcarboxylate interaction to decrease to a very low 2.516 (17) A. This is very short and it is possible that relief of the strain associated with this contact is responsible for a phase transition to a third form of serine as the pressure is increased from 7.3 to $8.1 \mathrm{GPa}$. In phase III the short OD $\cdots$ O hydroxylcarboxylate interaction is replaced by a bifurcated interaction in which the OD interacts with two carboxylate moieties with $\mathrm{O} \cdots \mathrm{O}$ distances of $2.638(15)$ and $2.775(13) \AA$. The trend towards opposite displacements of successive $C(5)$ chains formed by N1D5 ...O2 hydrogen bonds and a shortening of the distance between the chains continued through the II-toIII transition leading to very efficient packing of chains in phase III.

The Hirshfeld fingerprint plots show the changes in the balance of intermolecular interactions as a function of pressure. The increasing importance of D . . D interactions at high pressure is particularly apparent. Hydrogen bonds seem to be longer in L-serine-III than L-serine-II, and the increase in D...D interactions revealed in the fingerprint plots point to the compromise between hydrogen bonding and efficient packing that must be reached at elevated pressures.

We thank Dr Alan Coehlo for his advice on the use of TOPAS and Dr Josh McKinnon for his help with the program CrystalExplorer. We also thank EPSRC for funding and CCLRC for provision of neutron beam-time at ISIS.

\section{References}

Birch, F. (1947). Phys. Rev. 71, 809-824.

Boldyreva, E. V. (2004). NATO Science Series, II: Mathematics, Physics and Chemistry, edited by A. Katrusiak \& P. F. McMillan, Vol. 140, pp. 495-512. Dordrecht: Kluwer Academic Publishers.

Boldyreva, E. V., Ahsbahs, H. \& Weber, H.-P. (2003). Z. Kristallogr. 218, 231-236.

Boldyreva, E. V., Kolesnik, E. N., Drebushchak, T. N., Ahsbahs, H., Beukes, J. A. \& Weber, H.-P. (2005). Z. Kristallogr. 220, 58-65.

Bruno, I. J., Cole, J. C., Edgington, P. R., Kessler, M., Macrae, C. F., McCabe, P., Pearson, J. \& Taylor, R. (2002). Acta Cryst. B58, 389397.

Coelho, A. (2003). J. Appl. Cryst. 36, 86-95.

Coelho, A. (2005). Topas-A: General Profile and Structure Analysis Software for Powder Diffraction Data; http://members.optusnet.com.au/ alancoelho/.

Crystal Impact (2004). DIAMOND, Version 3.0. Crystal Impact GbR, Postfach 1251, 53002 Bonn, Germany. http://www.crystalimpact.com/diamond.

Dawson, A., Allan, D. R., Belmonte, S. A., Clark, S. J., David, W. I. F., McGregor, P. A., Parsons, S., Pulham, C. R. \& Sawyer, L. (2005). Cryst. Growth Des. 5, 1415-1427.

Fabbiani, F. P. A., Allan, D. R., Marshall, W. G., Parsons, S., Pulham, C. R. \& Smith, R. I. (2005). J. Cryst. Growth, 275, 185-192.

Farrugia, L. J. (1999). J. Appl. Cryst. 32, 837-838.

Fortes, A. D. (2004). PhD Thesis: Computational and Experimental Studies of Solids in the Ammonia-Water System. Department of Earth Sciences, University College, University of London.

Fourme, R., Kahn, R., Mezouar, M., Girard, E., Hoerentrup, C., Prange, T. \& Ascone, I. (2001). J. Synchrotron Rad. 8, 1149-1156.

Girard, E., Kahn, R., Mezouar, M., Dhaussy, A.-C., Lin, T., Johnson, J. E. \& Fourme, R. (2005). Biophys. J. 88, 3562-3571.

Hemley, R. J. \& Dera, P. (2000). Rev. Mineral. Geochem. 41, 335-419.

Johnson, F. H., Eyring, H. \& Stover, B. J. (1974). The Theory of Rate Processes in Biology and Medicine. New York: Wiley.

Katrusiak, A. (2004). NATO Science Series, II: Mathematics, Physics and Chemistry, edited by A. Katrusiak \& P. F. McMillan, Vol. 140, pp. 513-520. Dordrecht: Kluwer Academic Publishers.

Kistenmacher, T. J., Rand, G. A. \& Marsh, R. E. (1974). Acta Cryst. B30, 2573-2578.

Kolesnik, E. N., Goryainov, S. V. \& Boldyreva, E. V. (2005). Dokl. Phys. Chem. 404, 61-64.

Kuznetsov, A. Z., Dmitriev, V., Dubrovinsky, L., Prakapenka, V. \& Weber, H.-P. (2002). Solid State Commun. 122, 125-127. 
Leban, I. (1974). Cryst. Struct. Commun. 3, 237-239.

Marshall, W. G. \& Francis, D. J. (2002). J. Appl. Cryst. 35, 122125.

McKinnon, J. J., Spackman, M. A. \& Mitchell, A. S. (2004). Acta Cryst. B60, 627-668.

Miller, R. A. \& Schuele, D. E. (1969). J. Phys. Chem. Solids, 30, 589600.

Moggach, S. A., Allan, D. R., Morrison, C. A., Parsons, S. \& Sawyer, L. (2005). Acta Cryst. B61, 58-68.

Moggach, S. A., Allan, D. R., Parsons, S., Sawyer, L. \& Warren, J. E. (2005). J. Synchrotron Rad. 12, 598-607.
Moggach, S. A., Allan, D. R., Clark, S. J., Gutmann, M. J., Parsons, S., Pulham, C. R. \& Sawyer, L. (2006). Acta Cryst. B62, 296-309. Somero, G. N. (1992). Ann. Rev. Physiol. 54, 557-577.

Spek, A. L. (2003). J. Appl. Cryst. 36, 7-13.

Steiner, T. (2002). Angew. Chem. Int. Ed. 41, 48-76.

Tusvik, P. H., Mostad, A., Dalhus, B. \& Rosenqvist, E. (1999). Acta Cryst. C55, 1113-1115.

Waldorf, D. L. \& Alers, G. A. (1962). J. Appl. Phys. 33, 3266-3269.

Wolff, S. K., Grimwood, D. J., McKinnon, J. J., Jayatilaka, D. \& Spackman, M. A. (2005). CrystalExplorer1.5. University of Western Australia; http://www.Theochem.uwa.edu.au/crystal_explorer/. 